\title{
Original
}

\section{Idoneidad de tratamiento en sospechosos de glaucoma. Estudio de concordancia con el grupo de estudio RAND}

\author{
M.J. Fernández*, M.Á. Leal y J. Guzmán \\ Sección de Glaucoma, Hospital Virgen de La Salud, Toledo, España
}

\section{INFORMACIÓN DEL ARTÍCULO}

Historia del artículo:

Recibido el 31 de mayo de 2009

Aceptado el 18 de junio de 2010

Palabras clave:

Sospechosos de glaucoma

Hipertensión ocular

Factores de riesgo

Glaucoma de ángulo abierto

Presión intraocular

\section{R E S U M E N}

Objetivo: El grupo de estudio Appropriateness of Treating Glaucoma Suspects propone identificar qué pacientes sospechosos de glaucoma son apropiados o no para tratamiento. Nuestro objetivo es analizar la concordancia entre las decisiones clínicas de nuestra unidad de glaucoma y la del panel de expertos.

Métodos: Estudio retrospectivo de 77 pacientes (39 sospechosos, 38 hipertensos oculares). Se recogen datos de edad, antecedentes familiares, tamaño de disco óptico, cociente excavación/papila, presión intraocular, paquimetría y esperanza de vida. Se asignó una puntuación, a partir del esquema propuesto por el grupo de trabajo Appropriateness of Treating Glaucoma Suspects, según la cual se decide si es idóneo tratar o no hay consenso. Para analizar la concordancia se calculó el índice kappa.

Resultados: El valor de índice kappa fue 0,082 (intervalo de confianza al 95\% entre -0,11 y $0,27)$. Se obtuvo una concordancia completa en 41 de los sujetos $(63,08 \%)$. Nuestra decisión clínica fue tratar a 25 sujetos (22 no tratados según el comité de expertos) y no tratar a 40 (dos tratados por el comité de expertos). De los 12 individuos en los que el comité no encontraba consenso, en 8 nuestra actitud fue tratar.

Conclusiones: El índice de concordancia entre nuestra actitud y la del comité de expertos es bajo. Otras variables no recogidas por los expertos (campo visual y parámetros del láser confocal de barrido [Heidelberg Retina Tomography, HRT III]) fueron importantes en nuestras decisiones. No se han elaborado aún guías clínicas precisas en casos de sospecha por lo que es necesario individualizar el tratamiento.

( 2009 Sociedad Española de Oftalmología. Publicado por Elsevier España, S.L. Todos los derechos reservados.

${ }^{*}$ Autor para correspondencia.

Correo electrónico: mjfg05@gmail.com (M.J. Fernández). 


\title{
Appropriateness of treating glaucoma suspects. Concordancy study with the RAND study group
}

\author{
A B S T R A C T
}

Keywords:

Glaucoma suspect

Ocular hypertension

Risk factors

Open-angle glaucoma

Intraocular pressure

\begin{abstract}
Objective: The Appropriateness of Treating Glaucoma Suspects Study Group proposes to identify which patients suspected of glaucoma are suitable for treatment. Our objective is to analyse the concordance between our clinical decisions and this Study Group.

Methods: Retrospective study of 77 patients (39 suspects, 38 ocular hypertensives). Variables such as age, family history, intraocular pressure, disc size, cup-to-disc ratio, central corneal thickness and life expectancy were collected.They were scored following the Appropriateness of Treating Glaucoma Suspects Scale. Whether there was a consensus to treat or not depended on this value. The kappa index was used to measured the concordancy.

Results: The kappa index was 0.082 ( $95 \%$ confidence interval between -0.11 and 0.27 ). Total concordancy was obtained in 41 patients $(63,08 \%)$. We decided to treat 25 patients (22 of which were not treated by the experts) and not to treat 40 patients ( 2 of which were treated by the experts). In 12 patients there was noconsensus by the experts to treat, but we decided to treat 8 of them.

Conclussions: The concordancy index was low. Others variables such as visual fields and Heidelberg Retina Tomography (HRT III) parameters were not collected by the experts but were important in our decisions. There are no clinical guidelines on suspects. Individualised decisions are still necessary.
\end{abstract}

(C) 2009 Sociedad Española de Oftalmología. Published by Elsevier España, S.L. All rights reserved.

\section{Introducción}

Los pacientes sospechosos de glaucoma son definidos por las guías europeas de glaucoma como pacientes con campo visual y/o disco óptico y/o capa de fibras nerviosas normal o sospechosas, con al menos uno de estos requisitos sospechoso $^{1}$. Las guías oftalmológicas para decidir iniciar tratamiento en estos pacientes son complejas y dependen de factores oculares, sistémicos, médicos y psicosociales ${ }^{2}$. Por lo tanto, estos pacientes suscitan dudas a los expertos en glaucoma sobre la decisión de tratarlos o no. Existen múltiples estudios en los que se intenta evaluar los factores de riesgo de progresión a glaucoma en pacientes sospechosos ${ }^{3,4}$. El grupo de estudio Appropriateness of Treating Glaucoma Suspects en su trabajo publicado en $2009^{5}$ tiene como propósito identificar qué pacientes sospechosos de glaucoma son apropiados o no para tratamiento. El objetivo de nuestro trabajo es el análisis de la concordancia o acuerdo entre las decisiones clínicas de nuestra unidad de glaucoma y la del panel de expertos del estudio mencionado.

\section{Material y métodos}

Estudio retrospectivo en el que se incluyen mediante muestreo consecutivo 77 pacientes referidos a la Sección de Glaucoma del Hospital Virgen de la Salud. Del total, 39 fueron clasificados como sospechosos de glaucoma primario de ángulo abierto (GPAA) y 38 fueron clasificados como hipertensos oculares (HTO). Las exploraciones fueron realizadas de forma enmascarada por alguno de los cinco oftalmólogos de la Sección de Glaucoma del Hospital Virgen de la Salud. Se incluyó por defecto el ojo derecho de cada paciente o el izquierdo en caso de ser el único que cumplía criterios para ser considerado en el estudio.

En todos los pacientes incluidos en el estudio se recogieron datos de la historia clínica actual tanto oftalmológica como general. Los pacientes incluidos tenían un equivalente esférico de 5 dioptrías o menor y un defecto cilíndrico de 3 dioptrías o menor. Se excluyó a los pacientes que tenían factores de riesgo para desarrollar glaucoma secundario de ángulo abierto (seudoexfoliación, huso de Krukenberg, atrofia iridiana característica, antecedentes de uveítis o traumatismo ocular, signos de glaucoma corticoideo o aumento de la presión venosa epiescleral), así como los que cumplían criterios de glaucoma por cierre angular primario o secundario determinado mediante gonioscopia. Se clasificaron como HTO los individuos que cumplían los siguientes criterios: presión intraocular (PIO) mayor de $21 \mathrm{mmHg}$ en al menos dos ocasiones, con campo visual y exploración de la papila y de la capa de fibras nerviosas normales. Se clasificaron como sospechosos de glaucoma los individuos en que el aspecto de la papila era indicativo de GPAA, independientemente del valor de la PIO, con una exploración del campo visual normal o sospechosa.

Las imágenes del láser confocal de barrido (Heidelberg Retina Tomography, HRT III) con una calidad mejor o igual que aceptable (desviación estándar $\leq 30 \mu \mathrm{m}$ eran incluidas en el estudio; se rechazaban las que no cumplieran este criterio de calidad. De los parámetros estereométricos papilares se recogieron los correspondientes al diámetro excavación/ papila (E/P) así como el área del disco óptico. Se recogieron los datos de pérdida de varianza (LV) del segundo campo visual 
(octopus blanco-blanco, estrategia TOP) siempre que sus resultados fueran fiables (factor de fiabilidad < 15) y que el tiempo transcurrido entre la realización del campo visual y la exploración de la papila con HRT III fuera inferior a un mes. Los individuos que ya se encontraban tratados previamente a la realización de las pruebas diagnósticas recogidas no fueron incluidos en el estudio.

Se analiza la actitud que se tomó con esos pacientes (tratar o no), a partir de distintos factores de riesgo: edad, antecedentes familiares de primer grado diagnosticados de glaucoma.

Se asignó una puntuación, según el esquema propuesto por el grupo de trabajo que realizó el estudio Appropriateness of Treating Glaucoma Suspects, de la siguiente manera: edad: < 79 años, 0 puntos; > 79 años, 1 punto. Antecedentes familiares de primer grado con glaucoma: no, 0 puntos; sí, 1 punto. Tamaño del disco óptico: pequeño (< 1,6 mm), 1 punto; mediano (1,6-2,5 mm), 0 puntos; grande (> 2,5 mm), -1 punto (la medida del tamaño de la papila se tomó según los parámetros estereométricos del HRT III). Diámetro E/P: < 0,5, 0 puntos; 0,5-0,6, 1 punto; 0,7-0,8, 2 puntos; $\geq 0,9,4$ puntos (se utilizaron las medidas de la exploración biomicroscópica). PIO: $\leq 19 \mathrm{mmHg}$, 0 puntos; $20-23 \mathrm{mmHg}$, 2 puntos; $24-26 \mathrm{mmHg}$, 4 puntos; $27-29 \mathrm{mmHg}, 6$ puntos; $30-34 \mathrm{mmHg}, 8$ puntos. La medida de la PIO se tomó según el tonómetro de aplanación de Perkins. Paquimetría: < $520 \mu \mathrm{m}, 1$ punto; 520-580 $\mu \mathrm{m}$, 0 puntos; > $580 \mu \mathrm{m},-1$ punto. La medida del grosor de la córnea se tomó mediante el paquímetro IOPac Advanced (Heidelberg Engineering). La esperanza de vida: $\geq 90 \%$ de posibilidades de morir a 10 años, -2 puntos; < 90\% de posibilidades de morir a 10 años, 0 puntos. La esperanza de vida se calculó mediante el índice de Charlson, para estimar la supervivencia de vida a 10 años, calculando la mortalidad mediante la diferencia de uno menos la supervivencia.

En el total de puntuación se tomó como referencia el esquema propuesto por el grupo de trabajo que realizó el estudio Appropriateness of Treating Glaucoma Suspects, de la siguiente manera: con menos de 3 puntos totales no se consideró apropiado iniciar tratamiento antiglaucomatoso. Entre 3 y 6,5 puntos no existe consenso sobre la necesidad de trata- miento y por encima de 6,5 puntos se considera apropiado instaurar tratamiento. Para el análisis de la concordancia entre las decisiones clínicas tomadas en nuestro grupo de trabajo respecto a las recomendaciones del comité de expertos sólo se tuvo en cuenta a los individuos en los que, de acuerdo al consenso, se decidió tratar o no, excluyéndose aquellos para los que no había acuerdo sobre la idoneidad o no del tratamiento (entre 3 y 6,5 puntos totales).

En el análisis estadístico se utilizó el programa estadístico SPSS 10,1. Para valorar si existían diferencias significativas entre grupos se utilizaron la prueba de la t de Student y la prueba de la $\chi^{2}$. Para determinar la concordancia entre los datos del diámetro E/P determinado por biomicroscopia y HRT se calculó el coeficiente de correlación intraclase. Para valorar la concordancia entre las decisiones clínicas respecto al tratamiento tomadas por ambos grupos se calculó el índice kappa.

\section{Resultados}

Se incluyeron en el estudio los datos de 77 pacientes, 39 de ellos clasificados como sospechosos de GPAA $(50,6 \%)$ y 38 como HTO (49,4\%). Las tablas 1, 2 y 3 exponen la estadística descriptiva de la muestra.

De las variables recogidas existían diferencias estadísticamente significativas entre ambos grupos en relación con la LV y el diámetro E/P, tanto medido con HRT III como mediante oftalmoscopia (tabla 4). Estas tres variables fueron mayores en los individuos sospechosos $(p<0,05)$. Igualmente fue mayor el porcentaje de individuos sospechosos con antecedentes familiares de glaucoma $(p<0,05)$. Treinta y uno de los individuos presentaban antecedentes familiares de glaucoma, lo que representaba el $79,49 \%$ del total de sospechosos, frente al 31,58\% de los individuos con HTO (12 individuos). La PIO fue mayor en el grupo de HTO ( $p<0,05)$ respecto al de sospechosos (13 de los individuos tenían un valor de PIO mayor de $21 \mathrm{mmHg})$.

En el análisis de la concordancia entre la actitud clínica de tratar o no adoptada por nuestro grupo de estudio y la reco-

Tabla 1 - Variables descriptivas de la muestra

\begin{tabular}{|c|c|c|c|c|}
\hline & Grupo & $\mathrm{N}$ & Media & $\begin{array}{l}\text { Desviación } \\
\text { estándar }\end{array}$ \\
\hline \multirow[t]{2}{*}{ Edad } & Sospechoso & 39 & 60,92 & 11,98 \\
\hline & Hipertenso ocular & 38 & 57,71 & 13,57 \\
\hline \multirow[t]{2}{*}{ Pérdida de la varianza (LV) } & Sospechoso & 39 & 14,9818 & 11,0023 \\
\hline & Hipertenso ocular & 38 & 8,3864 & 7,3162 \\
\hline \multirow[t]{2}{*}{ Presión intraocular } & Sospechoso & 39 & 19,03 & 5,29 \\
\hline & Hipertenso ocular & 38 & 21,84 & 4,45 \\
\hline \multirow[t]{2}{*}{ Paquimetría } & Sospechoso & 39 & 550,85 & 33,57 \\
\hline & Hipertenso ocular & 38 & 556,34 & 27,35 \\
\hline \multirow[t]{2}{*}{ Diámetro excavación/papila medido por HRT } & Sospechoso & 39 & 0,3523 & 0,1668 \\
\hline & Hipertenso ocular & 38 & 0,2558 & 0,1463 \\
\hline \multirow[t]{2}{*}{ Diámetro excavación/papila medido por oftalmoscopia } & Sospechoso & 39 & 0,5367 & 0,1891 \\
\hline & Hipertenso ocular & 38 & 0,4026 & 0,1668 \\
\hline \multirow{2}{*}{$\begin{array}{l}\text { Puntos de riesgo de mortalidad medido con índice } \\
\text { de Charlson (\%) }\end{array}$} & Sospechoso & 39 & $-5,13$ & 0,32 \\
\hline & Hipertenso ocular & 38 & $-0,11$ & 0,45 \\
\hline
\end{tabular}


Tabla 2 - Variables descriptivas: antecedentes familiares de primer grado diagnosticados de glaucoma

\begin{tabular}{lccc}
\hline \multirow{2}{*}{ Grupo } & \multicolumn{2}{c}{ Antecedentes familiares } & \multirow{2}{*}{ Total } \\
\cline { 2 - 2 } & Sí & No & \\
\hline Sospechoso & 31 & 8 & 39 \\
Hipertenso ocular & 12 & 26 & 38 \\
Total & 43 & 34 & 77 \\
\hline
\end{tabular}

Tabla 3 - Variables descriptivas: tamaño del disco medido con HRT III

\begin{tabular}{lcc}
\hline & Frecuencia & $\%$ \\
\hline Pequeño $(<1,60)$ & 10 & 13 \\
Mediano $(\geq 1,6$ y $<2,5)$ & 58 & 75,3 \\
Grande $(>2,50)$ & 9 & 11,7 \\
Total & 77 & 100 \\
\hline
\end{tabular}

mendada por el comité de expertos según su sistema de puntuación, encontramos un valor de índice kappa de 0,082 global (intervalo de confianza [IC] del $95 \%$ entre $-0,11$ y 0,27), con una concordancia completa en 41 individuos (63,08\%). Para obtener dicho valor se excluyó a los individuos a los que al hallar la puntuación no existía consenso en la aplicación de tratamiento según el comité de expertos (12 individuos). Se decidió tratar a 25 sujetos, de los cuales en 22 no hubo concordancia con el comité de expertos, que decidió no tratar. De estos 22 individuos (9 HTO y 13 sospechosos), 15 tenían antecedentes familiares. Analizando por grupos, en el caso de los sospechosos de GPAA hubo acuerdo en la decisión terapéutica en 22 de los 35 casos analizados, mientras que en el caso de HTO hubo acuerdo en 19 de los 30 casos analizados. De los 12 individuos en los que el comité de expertos planteaba dudas sobre el beneficio del tratamiento, en 8 de ellos la actitud tomada en nuestro grupo de trabajo fue tratar (2 sospechosos y 6 HTO). De estos 8 individuos, 5 presentaban antecedentes familiares de glaucoma. De los 40 individuos en los que nuestra actitud clínica fue no tratar, en 2 de los individuos del grupo de HTO, aplicando las puntuaciones del comité de expertos, el total era de 7 puntos lo que aconsejaba trata- miento. Sin embargo, en estos individuos la actitud de nuestro grupo fue no tratar en ese momento debido a que la PIO no era excesivamente elevada y las pruebas funcionales y estructurales eran normales. Se decidió observación y seguimiento.

\section{Discusión}

El índice de concordancia entre nuestra actitud y la del comité de expertos es bajo. De los 25 casos que nosotros decidimos tratar sólo tres coinciden con la actitud de aquél (tabla 5).

En la mayoría de estos casos los pacientes son HTO y tienen antecedentes personales de glaucoma.

En la exploración de los pacientes sospechosos e HTO en nuestra práctica diaria incluimos la perimetría y el estudio de capas de fibras mediante HRT III. Por tanto, tenemos en cuenta más variables que las aquí recogidas y en algunos de estos sujetos los resultados muestran la posibilidad de que exista enfermedad, lo que apoya nuestra actitud de iniciar tratamiento.

Por otra parte, muchos de los sujetos a los que se decidió tratar presentaban antecedentes familiares de glaucoma. Podemos considerar que el conocimiento del glaucoma a través de familiares cercanos condiciona a la población en la necesidad no sólo de realizarse exploraciones para un diagnóstico precoz de la enfermedad, sino también en la preferencia por estar en tratamiento para así prevenir el daño sobre el nervio óptico. Es en estas circunstancias cuando pensamos que es esencial la entrevista con el paciente y dialogar sobre las posibilidades de iniciar o no tratamiento. Probablemente en este punto radique una de las grandes diferencias en nuestra muestra entre la decisión clínica adoptada y la aconsejada por el comité de expertos. En nuestra opinión, no es igual decidir sobre un supuesto teórico que frente a pacientes reales, que en ocasiones transmiten su preocupación, por lo que, en caso de estar indicado, tras una entrevista con ellos se decide pautar tratamiento.

Una situación similar encontramos en el grupo de pacientes sobre los que no existe consenso en cuanto al tratamiento según los expertos, y en los que nuestra opción fue tratar a la mayoría.

Tampoco midieron los panelistas la posibilidad o no de seguimiento por los pacientes ni sus factores psicosociales, que pueden hacer variar la actitud frente a un supuesto teórico.

Tabla 4 - Diferencias estadísticamente significativas entre grupos

\begin{tabular}{|c|c|c|c|}
\hline & \multirow[t]{2}{*}{$\mathrm{p}$} & \multicolumn{2}{|c|}{ Intervalo de confianza del 95\% } \\
\hline & & Mínimo & Máximo \\
\hline Edad & 0,274 & $-2,59$ & 9,02 \\
\hline Pérdida de la varianza (LV) & 0,048 & 5,96 & 13,1313 \\
\hline Presión intraocular & 0,014 & $-5,04$ & $-0,59$ \\
\hline Paquimetría & 0,434 & $-19,42$ & 8,43 \\
\hline Diámetro excavación/papila medido por HRT & 0,009 & 2,52 & 0,1678 \\
\hline Diámetro excavación/papila medido por oftalmoscopia & 0,002 & 5,30 & 0,2151 \\
\hline Puntos de riesgo de mortalidad medido con índice de Charlson (\%) & 0,547 & $-0,12$ & 0,23 \\
\hline
\end{tabular}


Tabla 5 - Actitud de nuestro hospital frente a la del comité de expertos

\begin{tabular}{lccc}
\hline & HVS & \multicolumn{2}{c}{ Comité de expertos } \\
\cline { 3 - 4 } & & $\begin{array}{c}\text { Con } \\
\text { tratamiento }\end{array}$ & $\begin{array}{c}\text { Sin } \\
\text { tratamiento }\end{array}$ \\
\hline Sospechoso & Con tratamiento 15 & 2 & 13 \\
Hipertenso & Sin tratamiento 20 & 0 & 20 \\
ocular & Con tratamiento 10 & 1 & 9 \\
& Sin tratamiento 20 & 2 & 18 \\
\hline Índice kappa 0,082 global (intervalo de confianza [IC] del 95\% \\
entre -0,11 y 0,27). HVS: Hospital Virgen de la Salud.
\end{tabular}

Son numerosos los estudios importantes sobre la necesidad de tratamiento en pacientes sospechosos como el Ocular Hypertension Treatment Study que objetiva la disminución del riesgo de desarrollar glaucoma en pacientes HTO (PIO entre 24 y $31 \mathrm{mmHg}$ ) al iniciar tratamiento ${ }^{6}$

A pesar de las diversas publicaciones que analizan los factores de riesgo asociados a glaucoma ${ }^{7,8}$, no se han elaborado aún guías clínicas precisas que nos indiquen claramente la necesidad de tratar a estos pacientes ${ }^{9}$. Es importante recalcar que nuestro estudio es limitado por su carácter retrospectivo y el tamaño muestral reducido.

La fina línea que separa en un individuo la sospecha del glaucoma de un glaucoma precoz es a menudo difícil de dilucidar. Son necesarios nuevos estudios que ayuden a aclarar la situación.

\section{B I B L I O G R A F Í A}

1. European Glaucoma Society. Terminology and guidelines for glaucoma. 3. ${ }^{a}$ edición. Savona: Dogma; 2008. p. 92.

2. American Academy of Ophthalmology Glaucoma Panel. Preferred Practice Pattern: primary open-angle glaucoma. American Academy of Ophthalmology. 2008;5:1-26.

3. Ocular Hypertension Treatment Study Group and European Glaucoma Prevention Study Group. Validated prediction model for the development of primary open-angle glaucoma in individuals with ocular hypertension. Ophthalmology. 2007; 114:10-9.

4. Friedmann DS, Wilson MR, Liebmann JM, Fechtner RD, Weinreb RN. An evidence-based assesment of risk factors for the progression of ocular hypertension and glaucoma. Am J Ophthalmol. 2004;138:S19-31.

5. Appropiatness of Treating Glaucoma Suspects RAND Study Group. For which glaucoma suspects is it appropriate to initiate treatment? Ophthalmology. 2009;116:710-6.

6. Kass MA, Heuer D, Higginbotham EJ, Johnson CA, Keltner JL, Miller JP, et al. The Ocular Hypertension Treatment Study. A randomized trial determines that topical ocular hypotensive medication delays or prevents the onset of primary open-angle glaucoma. Arch Ophthalmol. 2002;120:701-13.

7. European Galucoma Prevention Study (EGPS) Group. Predictive factors for open-angle glaucoma among patients with ocular hypertension in the European Glaucoma Prevention Study. Ophthalmology. 2007;114:3-9.

8. Gordon MO, Beiser JA, Brandt JD, Heuer DK, Higginbotham EJ, Johnson CA, et al. The Ocular Hypertension Treatment Study. Baseline factors that predict the onset of primary open-angle glaucoma. Arch Ophthalmol. 2002;120:714-20.

9. Lee BL, Wilson MR. Ocular hypertension treatment study (OHTS) commentary. Curr Opin Ophthalmol. 2003;14: 74-7. 